placed under-expanded stent and 11\% had severely calcified ISR. Procedural success was high (94\%), however, reasons for failure/abandonment included: (i) intra-procedural ischaemia with significant residual stenosis, (ii) prolonged procedural time, ischaemia and resistant stent under-expansion and (iii) prolonged procedural time, dissection with no-reflow, in the setting of a chronic total occlusion (CTO).

Complications Included Coronary dissection (13\%) of which the majority were successfully treated with conservative measures and coronary perforation (2\%) which was treated with prolonged balloon inflation. At a mean follow up of 10 months, 23\% underwent planned/staged-PCI, 4\% underwent CABG and there were $2(4 \%)$ deaths. There was a $51 \%$ reduction in CCS angina class from 2.9 (prior to IVL) to 1.4 (post IVL), $\mathrm{p}<0.00001$.

Conclusions The use of coronary IVL is a very effective percutaneous therapy for severe CAC. This cohort shows high procedural success with IVL and a significant reduction in CCS angina class at follow-up.

\section{CHARACTERISTICS OF CARDIAC ARREST SURVIVORS SUBSEQUENTLY DIAGNOSED WITH LONG QT SYNDROME ATTENDING AN INHERITED CARDIAC CONDITIONS CLINIC}

C Galvin, M Gallagher, H Cronin, T Prendiville, C McGorrian, J Galvin. Mater Misericordiae University Hospital/University College Dublin/Family Heart Screening Clinic, Dublin, Ireland

\subsection{6/heartjnl-2021-ICS.36}

Introduction/Background Within the Inherited Cardiac Condition (ICC)-affected population, Long QT Syndrome (LQTS) is the primary cause in $21 \%$ of aborted cardiac arrests (ACA) (Skinner et al. 2020). We reviewed the cases of ACA among the LQTS patient subgroup at an Irish tertiary referral centre for ICC.

Methods A retrospective study was undertaken to profile cases of ACA in LQTS probands attending the Family Heart Screening Clinic between February 2007 and June 2020. Relevant individuals were identified through the proband database and their records were used to attain information.

Results There were 656 LQTS patients of whom 212 were probands; and of whom 27 had an ACA prior to their diagnosis of LQTS. These were 17 females (mean age, 44 years) and 10 males (mean age, 27 years). Five had been taking QTprolonging drugs at the time of arrest while one was undergoing unascertained treatment for a RTI. Other precipitating factors included diarrhoeal illness $(n=1$; LQT1), swimming ( $n=2$; LQT1, LQT2), running $(n=1$; LQT3), undergoing alcohol detoxification $(n=1$; LQT1), initiating a bar fight $(n=1)$. Seventeen out of the $27(63.0 \%)$ had genetic testing. Eleven (64.7\% of gene-tested) had a Pathogenic (P) or Likely Pathogenic (LP) mutation detected; 2 of whom had a further VUS. Two (11.8\% of gene-tested) had VUSs alone and $4(23.5 \%$ of gene-tested) were gene negative. P and LP mutations occurred in SCN5A $(n=5), \operatorname{KCNQ} 1(n=4), \operatorname{KCNE} 1(n=1)$ and KCNH2 $(n=1)$. Our youngest affected patient was a young child when ACA occurred and had the highest burden of variants detected - a P variant in SCN5A and 3 VUSs in KCNE1, KCNE2, RYR2. Considerable variability in duration of QTc over time was noted in some patients (e.g. from $>600 \mathrm{~ms}$ to $<460 \mathrm{~ms}$ in the case of one proband). Of the 27 LQTS probands with ACA, 22 had ICDs implanted after the arrest. Of the probands who did not have ICD implanted, two had life-limiting complications associated with their arrest; a third fatally arrested in hospital after resuscitation from the initial ACA; a fourth had their ACA prior to widespread ICD implantation and has been successfully managed with Beta-Blockade alone. The fifth had been given 3 QT-prolonging drugs at the time of ACA and has been successfully managed with Beta-Blockade and QT-prolonging drug avoidance.

Conclusions This retrospective review of a single centre experience demonstrates genetic diagnosis in $65 \%$ of cases where sought in a post-ACA LQTS proband subgroup. Detection of $P$ and LP variants enables family screening for the affected gene. We saw increased prevalence of LQT3 among ACA sufferers; that QT-prolonging drug use was the most commonly recognised modifiable precipitator of cardiac arrest in this group. ICD insertion is generally undertaken in survivors of ACA with LQTS at this clinic.

\section{PREDICTIVE VALUE OF NORMAL HIGH-SENSITIVITY TROPONIN FOR SIGNIFICANT CORONARY ARTERY DISEASE IN NEW ATRIAL FIBRILLATION}

Z Butt, G Fitzgerald, F O'Herlihy, G O'Dea, A Casey, E McCrohan, S Quinn, K Hewitt, R Sheahan. Beaumont Hospital, Beaumont Rd, Dublin, D09V290, Ireland

\subsection{6/heartjnl-2021-ICS.37}

Background High-sensitivity troponin T (hsTNT) levels are often measured in patients who present with new-onset atrial fibrillation. The significance of an elevated hsTNT level in this clinical context has been examined at length. The implications of a normal hsTNT level in this scenario, meanwhile, are not as clearly understood.

Aims We aimed to examine the rate of significant coronary artery disease at angiography, or abnormal non-invasive investigations, in those patients with newly-diagnosed atrial fibrillation who presented with a normal high-sensitivity troponin. From this data we sought to describe the negative predictive value of a normal hsTNI for significant underlying coronary disease in this population.

Methods We examined all patients presenting with new-onset atrial fibrillation over a 6 -month period in a single centre after the adoption of a high-sensitivity troponin assay. We

Abstract 37 Table 1 Significant abnormality at coronary angiography or non-invasive ischaemic evaluation

\begin{tabular}{|l|l|}
\hline Coronary angiography & $\begin{array}{l}>50 \% \text { stenosis of left main stem } \\
>70 \% \text { stenosis of a major coronary } \\
\text { vessel } \\
30-70 \% \text { stenosis with an FFR }<0.8\end{array}$ \\
\hline Cardiac MRI & $\begin{array}{l}\geq 12 \% \text { of myocardium ischaemic, } \\
\text { and/or } \\
\geq 3 / 16 \text { segments with stress- } \\
\text { induced severe hypokinesis or } \\
\text { akinesis }\end{array}$ \\
\hline Echocardiography & $\begin{array}{l}\geq 3 / 16 \text { segments with stress- } \\
\text { induced severe hypokinesis or } \\
\text { akinesis }\end{array}$ \\
\hline $\begin{array}{l}>50 \% \text { stenosis in any major vessel } \\
\text { CT coronary }\end{array}$ &
\end{tabular}




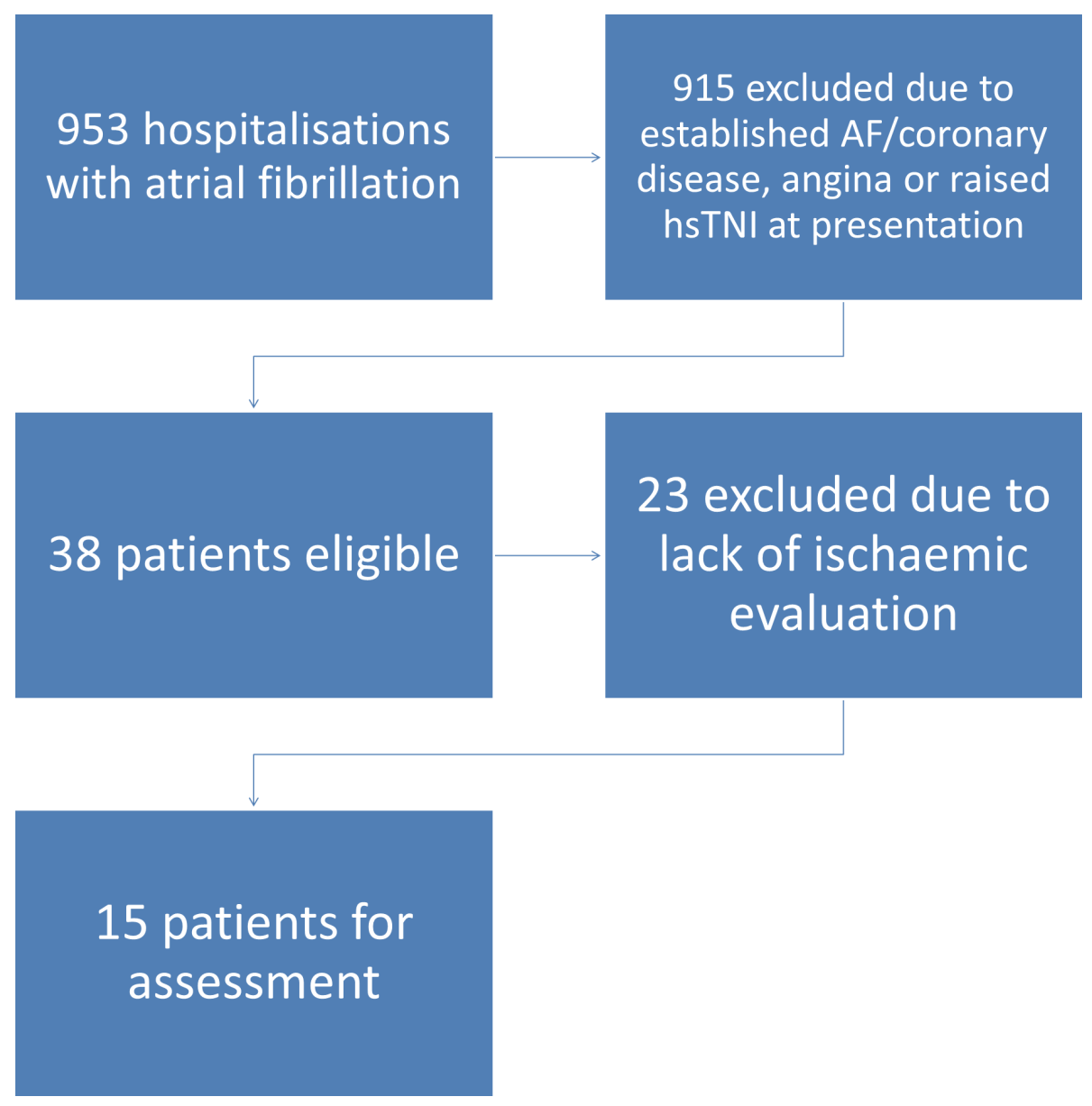

Abstract 37 Figure 1 Flow diagram demonstrating inclusion/exclusion criteria

identified patients who had hsTNT sent on the index presentation and who went on to have a subsequent ischaemic evaluation. We included invasive coronary angiography, CT coronary angiography, perfusion cardiac MRI, and dobutamine stress echocardiogram. We defined significant abnormalities on ischaemic assessment in accordance with accepted definitions in the literature (table 1). We examined 953 consecutive hospitalisations with atrial fibrillation over a 6-month period. Patients were initially excluded from our analysis if they had previously diagnosed atrial fibrillation or coronary artery disease, if they reported angina or if their hsTNT was elevated. We identified 38 patients suitable for assessment. 23 of these patients had not had an ischaemic evaluation at the time of our assessment, resulting in 15 patients for evaluation [figure 1]. Results All 15 patients presented with new-onset atrial fibrillation with rapid ventricular response. All ischaemic evaluations were performed within 9 months of initial presentation. 5/15 (33\%) patients presented with other primary illnesses with fast atrial fibrillation as a secondary feature. The mean HR at presentation was $135 \mathrm{bpm}(\mathrm{SD} \pm 20)$. 10/15 (66\%) of patients subsequently had invasive coronary angiography, while 4/15 (27\%) had a perfusion cardiac MRI and 1/15 (7\%) had a stress echocardiogram. 1 patient (7\%) satisfied the definition of significant coronary artery disease at invasive coronary angiography despite a normal hsTNT at presentation. Angiography revealed a chronic total occlusion of the RCA with non-obstructive disease in the LAD. Of note, this patient had presented without angina in atrial fibrillation with rates up to $120 \mathrm{bpm}$ for over two hours. The remaining $14 / 15$ patients
(93\%) had normal non-invasive assessments, normal coronary angiography or mild-moderate irregularities at coronary angiography that did not fit the criteria for significant coronary disease. We found that a normal hsTNT yielded a negative predictive value of $93 \%$ for significant coronary artery disease or abnormality at non-invasive assessment in those patients who went on to have such investigation.

Conclusion In patients with new-onset fast atrial fibrillation without angina, a normal high-sensitivity troponin is associated with a low incidence of significant coronary artery disease. Further work is required to examine the negative predictive value of a normal hsTNT level for significant coronary artery disease in larger patient populations and across multiple centres.

\section{8 \\ A YEAR IN THE LIFE OF VIRTUAL CLINIC FOR HEART FAILURE MANAGEMENT; A VALUABLE AID DURING THE COVID-19 PANDEMIC}

1,2,3B Wong, ${ }^{2} \mathrm{~J}$ Gallagher, ${ }^{1} \mathrm{~J} \mathrm{McC}$ ambridge, ${ }^{1} \mathrm{~A}$ Brennan, ${ }^{1,2,3} \mathrm{M}$ Ledwidge, ${ }^{4} \mathrm{~L}$ McCudden, 1,2,4 M Barrett, ${ }^{1} \mathrm{C}$ Halley, ${ }^{1,2,3} \mathrm{~K}$ McDonald. 'St Vincent's University Hospital, Dublin, Ireland; ${ }^{2}$ University College Dublin, Dublin, Ireland; ${ }^{3}$ Heartbeat Trust, Dublin, Ireland; ${ }^{4}$ Beacon Hospital, Dublin, Ireland

\subsection{6/heartjn|-2021-ICS.38}

Background Disseminating specialist knowledge into the community to facilitate care outside the hospital setting is an emerging focus of chronic disease management in Ireland and 